\title{
Eficacia del hierro aminoquelado en comparación con el sulfato ferroso como fortificante de un complemento alimentario en preescolares con deficiencia de hierro, Medellín, 2011
}

\author{
Maylen Liseth Rojas, Juliana Sánchez, Óscar Villada, Liliana Montoya, Alejandro Díaz, \\ Cristian Vargas, Javier Chica, Ana Milena Herrera \\ Grupo de Investigación Ciencias Básicas, Universidad CES, Medellín, Colombia
}

\begin{abstract}
Introducción. La disminución de los depósitos de hierro constituye el primer eslabón de la cadena conducente a la deficiencia de hierro, la carencia nutricional más prevalente y principal causa de anemia en todo el mundo, situación que puede prevenirse mediante la fortificación de alimentos.

Objetivo. Comparar la eficacia del hierro aminoquelado con el sulfato ferroso como fortificante de un complemento alimentario en preescolares con deficiencia de hierro.

Materiales y métodos. Se llevó a cabo un ensayo clínico triple ciego con distribución aleatoria de grupos. Se analizaron 56 preescolares con deficiencia de hierro (ferritina menor de $24 \mathrm{ng} / \mathrm{ml}$ ) a los que se les dio diariamente $13 \mathrm{~g}$ de leche con 12,5 mg de hierro,. Después de dos meses se midieron los niveles de hemoglobina, hematocrito y ferritina sérica.

Resultados. En el grupo con sulfato ferroso la concentración de ferritina sérica aumentó de 18,8 a 24,1 $\mathrm{ng} / \mathrm{ml}$, mientras que dicha variación fue de 18,4 a $29,7 \mathrm{ng} / \mathrm{ml}$ con el hierro aminoquelado, en ambos casos con diferencias significativas. El nivel de ferritina final difirió según el grupo de estudio, siendo mayor en el grupo con hierro aminoquelado $(p=0,022)$. La hemoglobina y el hematocrito no variaron después de la intervención. Las reacciones adversas en el grupo con sulfato ferroso fueron de 35,7\%, en contraste con el 42,9 \% en el grupo con hierro aminoquelado; cinco niños presentaron infección de las vías respiratorias, sin diferencias estadísticas.

Conclusiones. Los dos compuestos aumentan los niveles de ferritina, siendo mayor el aumento entre quienes toman leche con hierro aminoquelado. No fue diferente la incidencia de reacciones adversas o de infecciones entre los grupos.
\end{abstract}

Palabras clave: deficiencia de hierro, sulfato ferroso, preescolar. doi: http://dx.doi.org/10.7705/biomedica.v33i3.775

Effectiveness of iron amino acid chelate versus ferrous sulfate as part of a food complement in preschool children with iron deficiency, Medellín, 2011

Introduction: Iron depleted deposits are the first link in the chain of events leading to iron deficiency which is the most prevalent nutritional shortage and main cause of anemia worldwide. This situation can be prevented through food fortification.

Objective: To compare the efficacy of amino acid chelate iron with ferrous sulfate as fortifier of a dietary complement in preschoolers with iron deficiency.

Materials and methods: This study was a blinded clinical trial with randomized groups. We analyzed 56 preschoolers with iron deficiency (ferritin $<24 \mathrm{ng} / \mathrm{ml}$ ) that received $13 \mathrm{~g}$ of milk with $12.5 \mathrm{mg}$ of iron, either amino acid chelate or in the ferrous sulfate form. After two months, hemoglobin, hematocrit and serum ferritin concentrations were measured.

Results: In the ferrous sulfate group, ferritin concentration increased from $18.8 \mathrm{ng} / \mathrm{ml}$ to $24.1 \mathrm{ng} / \mathrm{ml}$, while the variation was of $18.4 \mathrm{ng} / \mathrm{ml}$ to $29.7 \mathrm{ng} / \mathrm{ml}$ in the amino acid chelate group, with statistically differences in both cases. Serum ferritin was different between groups, being higher in iron amino acid chelate group $(p=0.022)$. Hemoglobin and hematocrit levels did not change after the intervention. Adverse reactions in the ferrous sulfate group were $35.7 \%$, compared with $42.9 \%$ in the iron amino acid chelate group; 5 children had respiratory tract infection, without statistical differences.

Conclusions: Both compounds increased serum ferritin concentration, with a higher increase in those who were given milk with iron amino acid chelate. There were no differences in the adverse reactions and infections incidences between the groups.

\section{Contribución de los autores:}

Todos los autores participaron activamente en el diseño y ejecución del trabajo de investigación, además, colaboraron en la escritura del manuscrito

Identificador en Clinicaltrials.gov: NCT01412723 
Keywords: Iron deficiency, ferrous sulfate; child, preschool.

doi: http://dx.doi.org/10.7705/biomedica.v33i3.775

La Organización Mundial de la Salud (OMS) ha determinado que la deficiencia de hierro es uno de los problemas nutricionales de mayor magnitud en el planeta y una amenaza de gran importancia para la salud pública, presente tanto en países desarrollados como en desarrollo $(1,2)$. Solo durante el 2001 se calculó que en el mundo existían, aproximadamente, dos mil millones de personas anémicas -más del $30 \%$ de la población mundial-y que, de estas, cerca del $50 \%$ podrían atribuirse a la deficiencia de hierro $(3,4)$.

Inicialmente se presenta reducción de los depósitos de hierro en el cuerpo, momento en el que aún no se evidencian disfunciones causadas por los bajos niveles del mineral. Posteriormente, dicha disminución puede progresar hasta convertirse en deficiencia de hierro y, más adelante, causar anemia, principalmente a una temprana edad $(5,6)$.

La carencia de hierro se puede presentar en cualquier grupo de edad, aunque existen periodos de mayor vulnerabilidad como la infancia y la niñez, además de la edad fértil en el caso de las mujeres (7). Puede dar lugar a una baja resistencia a las infecciones, limitaciones en el desarrollo psicomotor, problemas en la función cognitiva, bajo rendimiento y fatiga, entre otros. Se encuentran casos en los que, a pesar de contar con un tratamiento oportuno y satisfactorio, las consecuencias persisten de manera irreversible durante toda la vida, específicamente cuando en población infantil la deficiencia de hierro avanza hasta convertirse en anemia (2).

Según los datos de mortalidad de la OMS, alrededor de 800.000 muertes al año podrían atribuirse a la deficiencia de hierro (aproximadamente, el 1,5 $\%$ del total) (4); además, reconoce la deficiencia de hierro como una de las principales causas de muerte en niños menores de cinco años (8).

Existen diferentes vías de solución y prevención para combatir la deficiencia de hierro, entre ellas, la fortificación de alimentos y el suplemento farmacológico han demostrado la mejor relación

\section{Correspondencia:}

Maylen Liseth Rojas, Edificio de Extensión de la Universidad de Antioquia, Calle $70 \mathrm{~N}^{\circ}$ 52-72, oficina 508, Medellín, Colombia Teléfono: (574) 2198900

maylenliseth@gmail.com

Recibido: 13/07/12; aceptado:14/03/13 costo-efectividad. Sin embargo, las desventajas que supone el suplemento, explicadas principalmente por el abandono de las intervenciones, producto de las reacciones adversas, hace de la fortificación la mejor estrategia (9).

Por otro lado, y a pesar de que con la fortificación las reacciones adversas disminuyen, no todas las reacciones indeseadas desaparecen, en gran parte debido a la usual y recomendada utilización del sulfato ferroso $\left(\mathrm{FeSO}_{4}\right)$ como compuesto químico en la fortificación de los alimentos (10); frecuentemente, esta se asocia a efectos adversos como náuseas, estreñimiento, dolor en el epigastrio, oscurecimiento de las heces y diarrea (11). El hierro aminoquelado (hierro quelado con glicina) aparece como una solución a este tipo de inconvenientes, debido a que estructuralmente es una forma de hierro no ionizable, con buena estabilidad química, que no cambia las propiedades organolépticas de los alimentos con que se mezcla (12), con una biodisponibilidad similar a la del sulfato ferroso, pero con una menor toxicidad en el aparato digestivo y una menor tasa de abandono de las intervenciones (12-14).

En el contexto colombiano, a pesar de las diferentes campañas ejecutadas contra la desnutrición y la anemia, la disminución de los depósitos de hierro continúa latente en la infancia; así, durante el 2005, a nivel nacional se observó una prevalencia de $47,9 \%$ de reducción de la concentración de hierro, en niños entre uno y cuatro años (15), quienes tenían un mayor riesgo de desarrollar anemia ferropénica.

En el caso específico de Medellín, no se ha desmostrado científicamente cuál compuesto para la fortificación con hierro, entre el hierro aminoquelado y el sulfato ferroso, presenta mayor eficacia para la normalización de los niveles de hierro en niños preescolares con depósitos disminuidos.

En esta investigación se comparó la eficacia del hierro aminoquelado con la del sulfato ferroso como fortificante de un complemento dietético, utilizando la leche como vehículo, en niños preescolares con deficiencia de hierro (ferritina menor de $24 \mathrm{ng} / \mathrm{dl}$ ), información que a la larga puede utilizarse a la hora de generar programas encaminados a mejorar las condiciones de salud de esta población.

\section{Materiales y métodos}

Se llevó a cabo un ensayo clínico triple ciego con asignación aleatoria de grupos, con intervención 
durante dos meses, en niños preescolares con deficiencia de hierro, inscritos en cuatro centros infantiles de una organización no gubernamental de Medellín.

La población inicial era de 379 niños. Después de aplicar los criterios de inclusión: edad entre 2 y 5 años inclusive, deficiencia de hierro (ferritina menor de $24 \mathrm{ng} / \mathrm{ml}$ ) y asistencia al centro infantil en jornada completa (8 horas), y los de exclusión: presencia de anemia (hemoglobina menor de $11 \mathrm{~g} /$ dl) y ausencia de análisis coprológico, se obtuvo una muestra por conveniencia de 64 niños repartidos entre los cuatro centros infantiles. La mitad de la muestra recibió $13 \mathrm{~g}$ de leche entera (Colanta®) fortificada con hierro aminoquelado y, los demás, leche con sulfato ferroso, de manera que de lunes a viernes recibieron $12,5 \mathrm{mg}$ de hierro esencial al día. La asignación de cada compuesto se hizo de manera aleatoria a los diferentes centros infantiles, utilizando bloques aleatorios de longitud variable. Al finalizar el estudio, debido a ocho pérdidas de seguimiento por cambio en el domicilio de los menores y por el consiguiente retiro de los niños de los centros infantiles, se analizó una muestra de 56 preescolares (figura 1).

La información se recolectó entre agosto y diciembre del 2011, con la participación de encuestadores estandarizados, cuidadoras del centro infantil (que reportaron las infecciones y reacciones adversas), y auxiliares de laboratorio que tomaron y procesaron las muestras sanguíneas y fecales.

En el análisis estadístico, primero se resumió la información de las variables continuas utilizando promedios con su correspondiente desviación

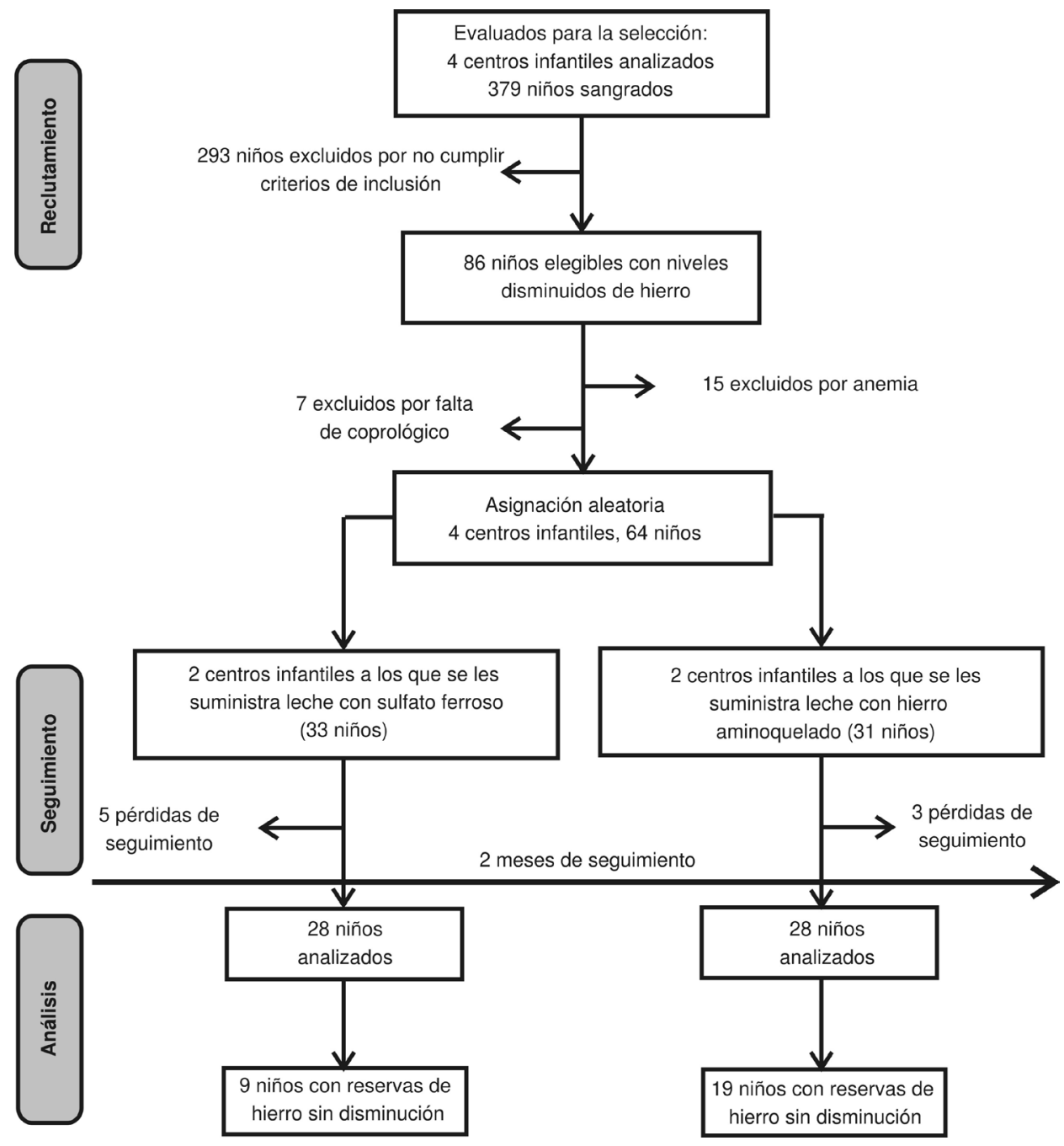

Figura 1. Diagrama de flujo del ensayo: eficacia del hierro aminoquelado en comparación con el sulfato ferroso como fortificante de un complemento alimentario en preescolares con deficiencia de hierro, Medellín, 2011 
estándar y las variables categóricas con proporciones; segundo, para la determinación de diferencias estadísticas entre la variable respuesta y las covariables, se utilizó la prueba t de Student para diferencia de promedios con varianzas iguales cuando se sustentó la normalidad o, en caso contrario, la prueba $U$ de Mann-Whitney. De igual manera, para analizar la asociación de las variables categóricas se usó la prueba de ji al cuadrado de independencia o, en su defecto, la prueba exacta de Fisher, dependiendo del número de frecuencias esperadas menores de cinco. La homogeneidad de cambios en las mediciones antes y después de los marcadores hematológicos, fue valorada con las pruebas de Wilcoxon y de McNeamar. En todos los casos, se usó una significancia estadística de $5 \%$.

Se valoró el estado nutricional antes de comenzar la intervención, utilizando las aplicaciones gratuitas de la de la OMS, la WHO Anthro, versión 3.2.2, de 2011 en el caso de los menores de cinco años y la WHO Anthro plus, versión 1.0.4, para los niños de cinco años o más (16). La información se digitalizó en una hoja de cálculo de MS Office Excel 2007; luego se exportó, almacenó y procesó en base de datos en el programa estadístico SPSS ${ }^{\mathrm{TM}}$, versión 19; para el cálculo de los intervalos de confianza y algunas pruebas de asociación, se utilizó la calculadora epidemiológica EpiDAT, versión 3.0. La presentación tabular, pictórica y textual, se hizo mediante el procesador de texto de MS Office Word 2007.

La investigación fue clasificada de riesgo mayor que el mínimo, y el protocolo fue evaluado y aprobado por el Comité de Ética de la Universidad CES mediante acta 041 de 2011. Se contó con el consentimiento informado y por escrito de los representantes legales de los preescolares; además, se pidió el asentimiento de los niños antes de tomar muestras sanguíneas. Todos los preescolares con diagnóstico de anemia por medio de exámenes de laboratorio, se remitieron a consulta médica.

\section{Resultados}

\section{Caracterización social y demográfica de los niños}

Se analizaron 56 niños (figura 1), con una mayor participación de niña $(57,14 \%)$ y una distribución idéntica por sexo entre los grupos de estudio. La mitad de los preescolares tenía 4,0 años o menos al comenzar la intervención $(\mathrm{Rq}=2,0)$, siendo mayores los preescolares que recibieron hierro aminoquelado ( $U$ de Mann Whitney, $p=0,027$ ).
Los niños provenían de familias con escasos recursos económicos $(41,4 \%$ recibían menos de un salario mínimo al mes), en su mayoría residían en viviendas de estrato uno (62,5\%), en situación de hacinamiento, entendido como la convivencia de más de tres personas por habitación, con un promedio de seis personas por vivienda y una desviación estándar (DE) de 2,0. Los niños también pertenecían a familias con miembros de distintas generaciones en la misma residencia, con padres con estudios de secundaria, que convivían en unión libre, con madres cabeza de la familia $(32,1 \%)$, de las cuales, muchas habían sufrido procesos de desplazamiento forzoso $(30,4 \%)$. Cuando se consideró el compuesto fortificante, no se encontraron diferencias estadísticas, a excepción de los ingresos que percibían mensualmente en el hogar, siendo estos mayores entre los que tomaron leche con sulfato ferroso $\left(\chi^{2}\right.$ de independencia, $\mathrm{p}=0,029$ ) (cuadro 1).

\section{Parasitosis}

Antes de comenzar el estudio, la proporción de parasitosis fue de $57,1 \%$ para los niños que recibieron sulfato ferroso y de $53,6 \%$ para los que recibieron hierro aminoquelado, sin diferencias estadísticas $\left(\chi^{2}\right.$ de independencia de Pearson, $p=0,419)$. Después de la intervención solo fue posible conocer el estado parasitario del $66,1 \%$ de los niños. La prevalencia final de parasitosis en cada grupo fue de $32,1 \%$, sin diferencias estadísticas $\left(\chi^{2}\right.$ de independencia de Pearson, $p=1,000)$.

Tampoco variaron las proporciones de parasitosis antes y después de la intervención dentro de cada grupo de estudio (McNemar, sulfato ferroso $p=0,289$; hierro aminoquelado $p=1,000$ ).

\section{Niveles de hemoglobina, hematocrito y ferritina sérica}

La línea basal de los marcadores hematológicos fue similar entre los grupos. Al finalizar la intervención, los niveles de hemoglobina y hematocrito no fueron diferentes, pero sí lo fue el de ferritina, siendo mayor entre los que recibieron hierro aminoquelado ( $U$ de Mann Whitney, $p=0,022$ ) (cuadro 2). No se encontraron diferencias en el perfil hematológico según la presencia de parásitos antes o después de la intervención ( $U$ de Mann Whitney, $p=0,069$ y $p=0,095)$.

Se analizó también la evolución del perfil hematológico dentro de los grupos. En ninguno de ellos se evidenciaron cambios cuando al comparar los 
Cuadro 1. Distribución de frecuencias de las características sociales y demográficas de los niños preescolares con deficiencia de hierro, según grupo de estudio

\begin{tabular}{|c|c|c|c|c|}
\hline & Sulfato ferroso & Hierro aminoquelado & Total & p \\
\hline \multicolumn{5}{|l|}{ Sexo } \\
\hline Hombre, n (\%) & $12(42,86)$ & $12(42,86)$ & $24(42,86)$ & $1,000^{*}$ \\
\hline Mujer, n (\%) & $16(57,14)$ & $16(57,14)$ & $32(57,14)$ & \\
\hline \multicolumn{5}{|l|}{ Edad (años) } \\
\hline $\bar{X} \pm D E$ & $3,50(1,07)$ & $4,17(0,86)$ & $3,83(1,02)$ & $0,027^{\star *}$ \\
\hline $\mathrm{Me}(\mathrm{Rq})$ & $3,29(2,05)$ & $4,35(1,39)$ & $4,02(2,04)$ & \\
\hline $\mathrm{CV} \%$ & $30,57 \%$ & $20,62 \%$ & $26,63 \%$ & \\
\hline \multicolumn{5}{|l|}{ Estrato socioeconómico de la vivienda } \\
\hline Estrato 1, n (\%) & $14(50,00)$ & $21(75,00)$ & $35(62,5)$ & $0,053^{*}$ \\
\hline Estrato $2-3^{\dagger}, \mathrm{n}(\%)$ & $14(50,00)$ & $7(25,0)$ & $21(37,5)$ & \\
\hline \multicolumn{5}{|l|}{ Tipo de vivienda } \\
\hline $\mathrm{Casa}^{\ddagger}, \mathrm{n}(\%)$ & $18(64,29)$ & $16(57,14)$ & $34(60,71)$ & -- \\
\hline Apartamento, n (\%) & $7(25,00)$ & $6(21,43)$ & $13(23,21)$ & $0,956^{*}$ \\
\hline Cuarto - Otro ${ }^{\dagger}, \mathrm{n}(\%)$ & $3(10,71)$ & $6(21,43)$ & $9(16,07)$ & $0,457^{\S}$ \\
\hline \multicolumn{5}{|l|}{ Madre cabeza de familia } \\
\hline Sí, n (\%) & $8(28,57)$ & $10(35,71)$ & $18(32,14)$ & $0,567^{*}$ \\
\hline No, n (\%) & $20(71,43)$ & $18(64,29)$ & $38(67,86)$ & \\
\hline \multicolumn{5}{|l|}{ Ingresos familiares en el último mes } \\
\hline Menos de un SMMLV, n (\%) & $7(25,00)$ & $16(57,14)$ & $23(41,07)$ & $0,029^{*}$ \\
\hline Un SMMLV y más, n (\%) & $21(75,00)$ & $12(42,86)$ & $33(58,93)$ & \\
\hline \multicolumn{5}{|l|}{ Tipo de afiliación del niño al SGSSS } \\
\hline Contributivo, $\mathrm{n}(\%)$ & $4(14,29)$ & $2(7,14)$ & $6(10,71)$ & $0,670^{\S}$ \\
\hline Subsidiado, n (\%) & $24(85,71)$ & $26(92,86)$ & $50(89,29)$ & \\
\hline \multicolumn{5}{|l|}{ Escolaridad de la madre } \\
\hline Ninguna, $\mathrm{n}(\%)$ & $2(7,14)$ & $1(3,57)$ & $3(5,36)$ & $1,000^{\S}$ \\
\hline Primaria, n (\%) & $7(25,00)$ & $8(28,57)$ & $15(26,79)$ & $0,827^{*}$ \\
\hline Secundaria - técnica ${ }^{\dagger \ddagger}, \mathrm{n}(\%)$ & $19(67,86)$ & $19(67,86)$ & $38(67,86)$ & -- \\
\hline \multicolumn{5}{|l|}{ Población desplazada } \\
\hline Sí, n (\%) & $6(21,43)$ & $11(39,29)$ & $17(30,36)$ & $0,245^{*}$ \\
\hline No, n (\%) & $22(78,57)$ & $17(60,71)$ & $39(69,64)$ & \\
\hline
\end{tabular}

Me: mediana; Rq: rango intercuartil; CV: coeficiente de variación; SMMLV: salario mínimo mensual legal vigente; SGSSS: Sistema General de Seguridad Social en Salud

"Prueba de ji al cuadrado de independencia de Pearson

"Prueba U de Mann-Whitney

+ Se unieron las dos categorías dado que en algunas de ellas existen frecuencias esperadas menores de 5

₹ Categoría de referencia

$\S$ Prueba exacta de Fisher

Cuadro 2. Perfil hematológico antes y después de la intervención, entre grupos de estudio

\begin{tabular}{|c|c|c|c|c|c|}
\hline & \multicolumn{2}{|c|}{ Sulfato ferroso } & \multicolumn{2}{|c|}{ Hierro aminoquelado } & \multirow[t]{2}{*}{$\mathbf{P}$} \\
\hline & $\bar{X} \pm(D E)$ & $\operatorname{Me}(\mathbf{R q})$ & $\bar{X} \pm(D E)$ & $\operatorname{Me}(\mathbf{R q})$ & \\
\hline \multicolumn{6}{|l|}{ Antes } \\
\hline Hemoglobina & $11,50(2,30)$ & $11,75(0,60)$ & $11,91(0,56)$ & $12,05(1,15)$ & $0,712^{* *}$ \\
\hline Hematocrito & $34,01(6,83)$ & $35,2(2,43)$ & $35,6(2,01)$ & $36,00(3,63)$ & $0,294^{*}$ \\
\hline Ferritina & $18,75(3,54)$ & $19,14(6,51)$ & $18,40(3,97)$ & $18,95(6,05)$ & $0,723^{+\dagger}$ \\
\hline \multicolumn{6}{|l|}{ Después } \\
\hline Hemoglobina & $11,17(0,93)$ & $12,20(1,25)$ & $11,97(0,61)$ & $11,95(1,00)$ & $0,344^{\dagger \dagger}$ \\
\hline Hematocrito & $35,65(2,53)$ & $35,75(2,93)$ & $35,31(1,92)$ & $35,1(2,78)$ & $0,987^{*}$ \\
\hline Ferritina & $24,08(10,02)$ & $22,61(10,46)$ & $29,74(11,49)$ & $26,30(15,50)$ & 0,022 \\
\hline
\end{tabular}

Me: mediana; Rq: rango intercuartil

* Prueba U de Mann-Whitney

t† Prueba t de Student para diferencia de medias independientes con varianzas iguales 
niveles de hemoglobina y hematocrito después de la intervención, con respecto a los encontrados antes de complementar con hierro; fue diferente con el nivel de ferritina, que fue significativamente diferente tanto en el grupo con sulfato ferroso como en aquel con hierro aminoquelado (prueba de Wilcoxon, $p=0,003$ para sulfato ferroso y $p=0,000$ para hierro aminoquelado) (figura 2)

Después de dos meses de complemento alimentario, se observó una disminución en la deficiencia de hierro, siendo mayor la proporción de niños en que se normalizaron sus depósitos a partir del consumo de hierro aminoquelado (67,9 \%), en comparación con quienes tomaron sulfato ferroso $(32,1 \%)$ (cuadro 3).

El riesgo de continuar con niveles disminuidos en el grupo que recibió sulfato ferroso fue de 0,68 y, en el del hierro aminoquelado, de 0,32 (riesgo relativo de 0,$\left.47 ; \mathrm{IC}_{95 \%} 0,261-0,859\right)$; es decir, el riesgo de tener disminución de los niveles de hierro después de consumir durante dos meses leche fortificada con sulfato ferroso, es el doble del riesgo entre quienes toman leche enriquecida con hierro aminoquelado. La reducción del riesgo fue de 52,6 \%, la diferencia de los riesgos fue de 0,36 (reducción absoluta del riesgo, RAR) y el número necesario de tratar de $2,8(\mathrm{NNT}) \approx 3$ preescolares).

\section{Estado nutricional}

Antes de comenzar la intervención, el 18,4 \% de los niños presentó retraso en la talla para la edad; sin embargo, no se encontraron diferencias en este indicador entre los grupos de estudio (figura 3). Al considerar el peso para la talla, el 83,9\%

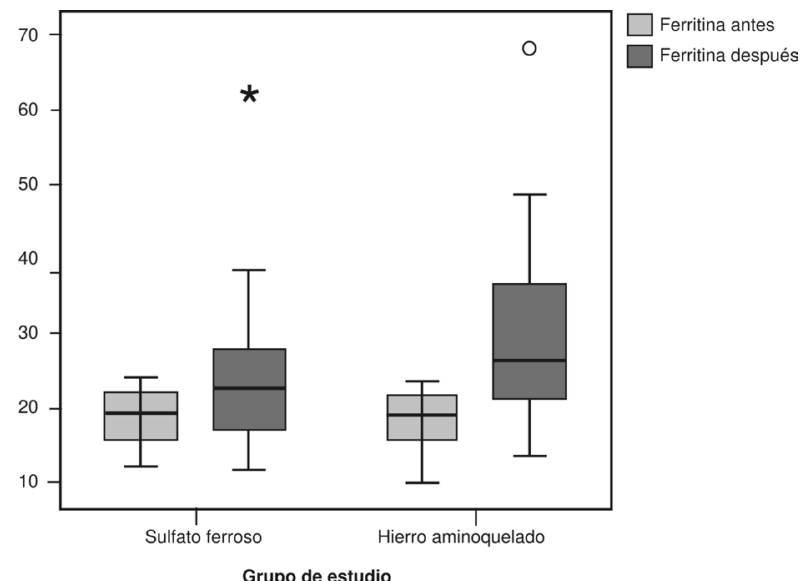

Figura 2. Nivel de ferritina sérica antes y después de la intervención, según grupo de estudio

de los niños presentaba un adecuado estado nutricional, en contraste con el 16,1\% adicional, niños con malnutrición (riesgo de bajo peso, sobrepeso u obesidad); no hubo diferencias según el grupo (prueba exacta de Fisher, $p=1,000$ ). Según el estado nutricional, tanto en los niños con malnutrición, como en quienes tenían un peso adecuado para la talla, aumentaron los niveles de ferritina después de la intervención (Wilcoxon para niños con peso adecuado, $p=0,000$ y t de Student para diferencia de medias relacionadas para niños con malnutrición, $p=0,038$ ).

\section{Infecciones según grupo de estudio}

Se reportó la presencia de infección de las vías respiratorias en el $8,6 \%$ de los casos, el $80 \%$ de los cuales recibía leche con hierro aminoquelado; sin embargo, estas diferencias no fueron significativas $\left(\chi^{2}\right.$ de independencia, $p=0,372$ ).

Cuadro 3. Niveles hematológicos antes y después de la intervención, dentro de los grupos de estudio

\begin{tabular}{|c|c|c|c|c|c|c|}
\hline & \multicolumn{2}{|c|}{ Sulfato ferroso } & \multicolumn{4}{|c|}{ Hierro aminoquelado } \\
\hline & Antes & Después & p & Antes & Después & $\mathbf{P}$ \\
\hline \multicolumn{7}{|l|}{ Hemoglobina } \\
\hline $\bar{x}(\mathrm{DE})$ & $11,50(2,30)$ & $11,17(0,93)$ & \multirow{3}{*}{$0,211 \S \S$} & $11,91(0,56)$ & $11,97(0,61)$ & \multirow{3}{*}{$0,582^{n+*}$} \\
\hline & & & & & & \\
\hline $\mathrm{Me}(\mathrm{Rq})$ & $11,75(0,60)$ & $12,20(1,25)$ & & $12,05(1,15)$ & $11,95(1,00)$ & \\
\hline \multicolumn{7}{|l|}{ Hematocrito } \\
\hline $\bar{x} \bar{x}(D E)$ & $34,01(6,83)$ & $35,65(2,53)$ & \multirow{3}{*}{$0,498^{* * *+}$} & $35,6(2,01)$ & $35,31(1,92)$ & \multirow{3}{*}{$0,682 \S \S$} \\
\hline & & & & & & \\
\hline $\mathrm{Me}(\mathrm{Rq})$ & $35,2(2,43)$ & $35,75(2,93)$ & & $36,00(3,63)$ & $35,1(2,78)$ & \\
\hline \multicolumn{7}{|l|}{ Ferritina } \\
\hline $\bar{x} \bar{x}(\mathrm{DE})$ & $18,75(3,54)$ & $24,08(10,02)$ & \multirow{3}{*}{$0,003 \S \S$} & $18,40(3,97)$ & $29,74(11,49)$ & \multirow{3}{*}{$0,000 \S$} \\
\hline & & & & & & \\
\hline $\mathrm{Me}(\mathrm{Rq})$ & $19,14(6,51)$ & $22,61(10,46)$ & & $18,95(6,05)$ & $26,30(15,50)$ & \\
\hline
\end{tabular}

Me: mediana; Rq: rango intercuartil

$\S$ Prueba de Wilcoxon

*** Prueba t de Student para diferencia de medias relacionadas 


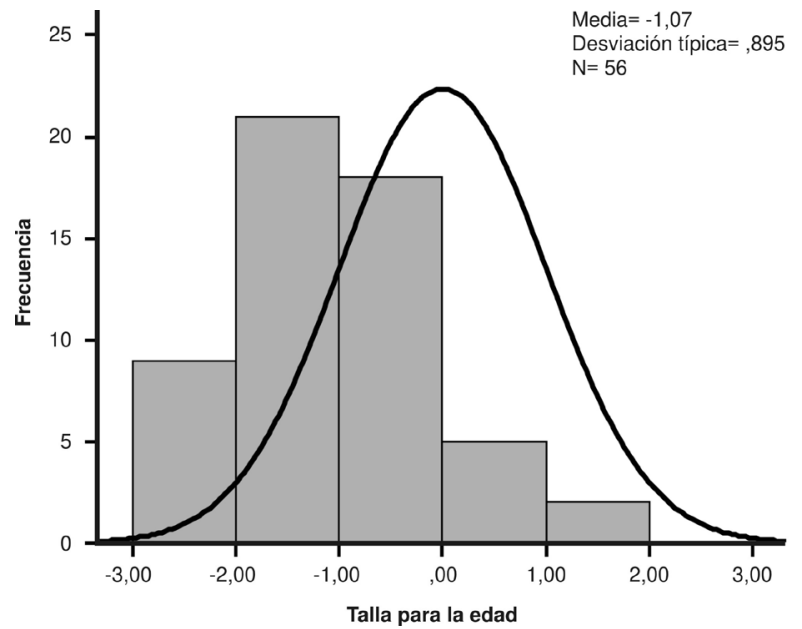

Figura 3. Distribución de desviaciones estándar de la talla para la edad en los preescolares menores de cinco años

\section{Reacciones adversas a la ingestión del alimento fortificado}

El $39,3 \%$ de los niños presentaron, al menos, una reacción adversa durante el periodo de observación. La proporción fue más alta entre los que tomaron hierro aminoquelado; no obstante, no se encontraron diferencias estadísticas $\left(\chi^{2}\right.$ de independencia, $p=0,584$ ) (cuadro 4).

\section{Discusión}

En este estudio se presenta el experimento comparativo de los compuestos hierro aminoquelado y sulfato ferroso, como complementos alimentarios en niños preescolares, de dos a cinco años con disminución de los niveles de hierro, utilizando la leche como vehículo fortificado.

Sus resultados muestran una reducción en la proporción de niños con disminución de los niveles de hierro después de dos meses de complemento alimentario, siendo mayor la proporción de aquellos en que se normalizaron sus depósitos a partir del consumo de leche con hierro aminoquelado..

Estos efectos no son solo significativos estadísticamente, sino también importantes en los aspectos biológico, clínico y social. En lo biológico, se disminuye el riesgo de que los niños presenten anemia y, en la práctica clínica, se demuestra un aumento en los niveles de ferritina en sangre a partir de estrategias no farmacológicas en el período estudiado. En lo social, además de entender la salud como un bien, se ha encontrado que la deficiencia de hierro causa pérdidas económicas a las sociedades en términos de crecimiento, desarrollo y productividad, al generar adultos con limitaciones físicas e intelectuales, con bajos niveles de productividad y elevadas tasas de enfermedades crónicas e incapacitantes $(2,10,17)$.

Se evidenció un incremento de la ferritina sérica en los dos grupos de estudio, de manera que ambos compuestos incidieron positivamente en la variable que indica la cantidad de hierro en los depósitos corporales. Dicha diferencia fue mayor entre quienes recibieron una dieta enriquecida

Cuadro 4. Distribución de las reacciones adversas al consumo de la leche fortificada según grupo de estudio

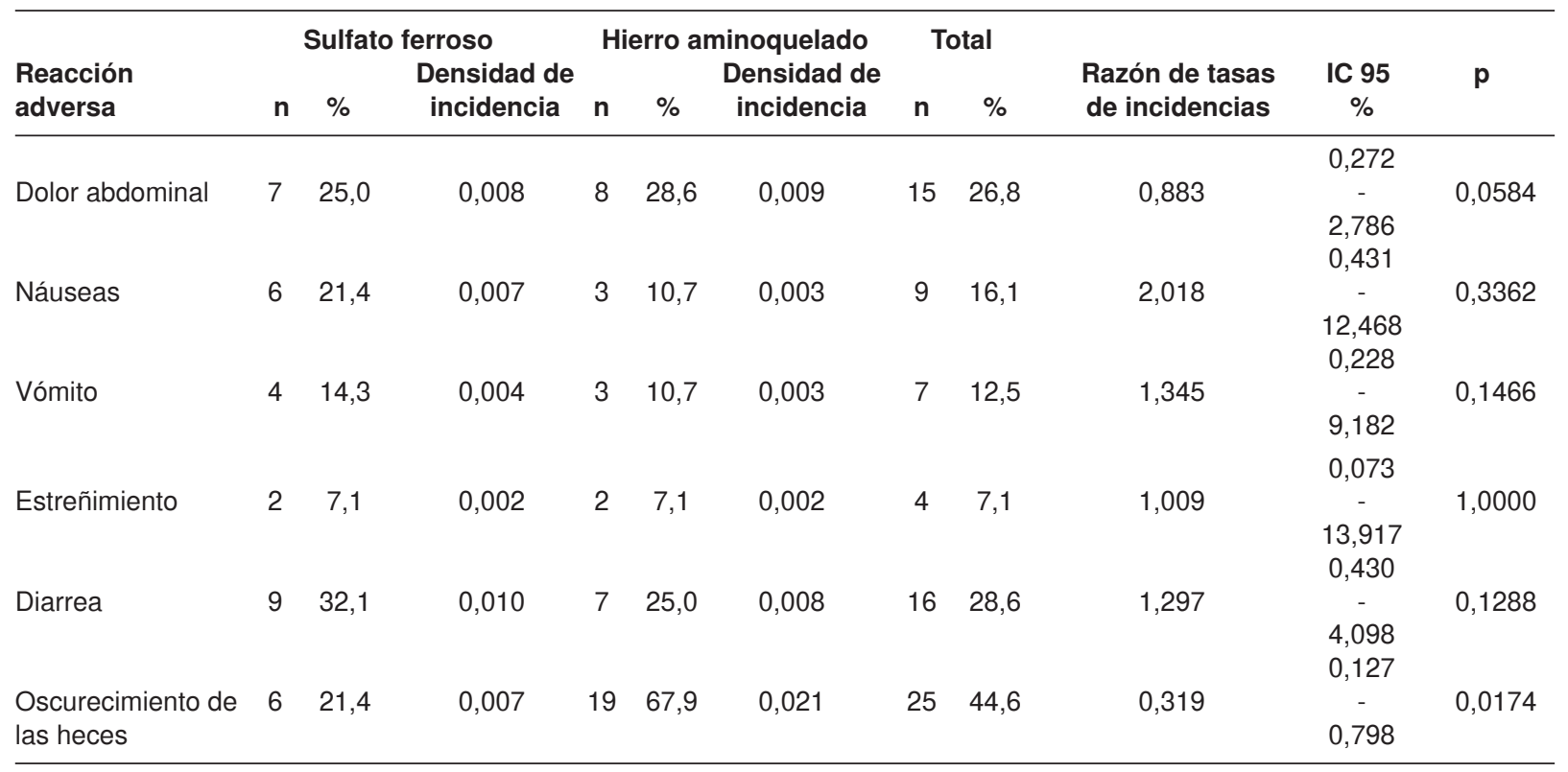


con hierro aminoquelado, mientras que los niños que recibieron dicho compuesto presentaron dos veces la probabilidad de normalizar sus niveles de ferritina sérica con respecto a los preescolares que recibieron sulfato ferroso.

Como se informó en el apartado de resultados, no se encontró asociación entre el compuesto de hierro y los niveles de hemoglobina y hematocrito, aunque algunos niños desarrollaron anemia y otros disminuyeron sus niveles de hematocrito, situación que puede explicarse por alteraciones no ferropénicas, en este caso de causa desconocida, que para ser descubierta requeriría estudios adicionales que desbordan el objetivo de esta investigación.

El hallazgo principal de este estudio concuerda con algunas investigaciones donde reportan un mayor efecto del hierro aminoquelado que del sulfato ferroso, con una absorción de dos a cuatro veces más alta; incluso, los resultados coinciden con estudios de diseño metodológico diferente, en que se utilizaron otros vehículos o se estudiaron los compuestos como suplementos nutricionales (18-20).

En un estudio diseñado para evaluar la biodisponibilidad del hierro aminoquelado en leche entera, los autores observaron que la absorción del hierro a partir de $3 \mathrm{mg}$ de hierro por litro fue de 2 a 2,5 veces mayor que la observada con leche fortificada de manera similar pero con sulfato ferroso $(21,22)$. Así, los resultados del estudio concuerdan en que por cada niño en que se normalizaron los depósitos de hierro con sulfato ferroso, en dos esto se logró con el hierro aminoquelado, lo cual sustenta la comparación ya que los estudios compartían el vehículo fortificado y los compuestos comparados.

En contraposición, Mayumi, et al., en el 2008, concluyeron que el sulfato ferroso es más eficaz, al suministrar diariamente y durante cuatro meses hierro aminoquelado y sulfato ferroso a 18 pacientes con gastrectomía para el tratamiento de anemia por deficiencia de hierro; al finalizar el estudio, los pacientes tratados con hierro aminoquelado no aumentaron sus niveles de hemoglobina y ferritina, a diferencia de aquellos a quienes se les suministró sulfato ferroso, con aumento en ambos marcadores; del grupo con sulfato ferroso, solo un paciente continuó anémico, en comparación con seis de los que recibieron hierro aminoquelado (23). Sin embargo, esta diferencia podría explicarse por los procesos de absorción de los minerales en este tipo de pacientes y por la estrategia utilizada, ya que no se fortificó un alimento sino que se administró un suplemento.

En el estudio de Fox, et al. en Inglaterra, niños de nueve meses de edad recibieron vegetales en papilla fortificados con hierro aminoquelado o sulfato ferroso, sin que se encontraran diferencias estadísticas en la absorción de hierro entre los grupos estudiados, de manera que ninguno aumentó los niveles de ferritina en los niños (13). Este resultado difiere también del hallado en esta investigación, lo que podría explicarse por el vehículo utilizado. Se debería estudiar con mayor profundidad el comportamiento del hierro aminoquelado cuando se fortifican otros alimentos con inhibidores de la absorción del hierro, como los fitatos.

En cuanto a la población estudiada, las condiciones de los niños incluidos permitió de alguna manera, no solo contar con grupos comparables en cuanto a sus características demográficas, económicas, sociales, parasitarias, nutricionales y hematológicas, sino también, controlar de manera natural las posibles diferencias en la calidad de la dieta de los niños por fuera del centro infantil, pues la mayoría de los preescolares solo recibían alimentación en las instalaciones de la Fundación de Atención a la Niñez.

En cuanto a la edad, si bien la mediana fue mayor entre los que recibieron hierro aminoquelado que en quienes tomaron sulfato ferroso, la distribución fue producto del azar y no compromete la comparabilidad de los grupos pues en ambos casos se trataba de preescolares.

Como en otros estudios, antes de comenzar con el complemento alimentario, los niños recibieron 10 $\mathrm{ml}$ de albendazol en suspensión (23) para controlar la interacción reportada en la literatura científica entre la presencia de parásitos y la absorción del hierro, en la que el parásito puede actuar de dos maneras: causando pérdida sanguínea intestinal o compitiendo por el consumo de las sustancias que ingiere la persona $(4,24)$. Sin embargo, antes de aplicar la desparasitación se hizo el análisis coprológico, igual que al finalizar la intervención, con el propósito de controlar y analizar la posible interacción durante el análisis de los resultados.

Se encontró que, si bien la prevalencia de parasitosis intestinal fue igual antes y después de la intervención, el análisis debe hacerse con prudencia en consideración de la imposibilidad de 
conocer el estado parasitario del 33,9\% de los niños y de la función del antiparasitario utilizado que erradica básicamente helmintos (uncinarias, Ascaris lumbricoides y tricocéfalos), los cuales pueden producir sangrado microscópico y deficiencia de hierro. En los análisis coprológicos finales no se encontró este tipo de parásitos, por lo que la desparasitación podría considerarse efectiva.

En cuanto al estado nutricional, la distribución de las desviaciones estándar en el indicador talla para la edad, comparada con la distribución de la población de referencia ofrecida por la OMS en el 2006 (25), mostró un desplazamiento de la curva hacia la izquierda, lo cual indica que un porcentaje mayor al esperado de niños incluidos en el estudio presentó retraso en la talla.

En la distribución normal ofrecida por la OMS, se esperaría que, aproximadamente, el 13,4\% de los preescolares presentara riesgo de baja talla para la edad (-2 DE > talla/edad < -1 DE) $(8,16,25)$; esta situación se evidenció en $34,7 \%$ de los preescolares, lo que posiblemente se deba al tipo de población beneficiaria de la Fundación de Atención a la Niñez, al tratarse de niños residentes en algunos de los barrios y sectores más marginados de Medellín (26), quienes en su mayoría no reciben otros alimentos fuera de la institución.

Una de las justificaciones para tomar el sulfato ferroso como el compuesto suministrado al grupo control, fue su conocida recomendación a la hora de implementar programas de fortificación de alimentos. Dicha recomendación se basa en la relación costo-beneficio y en su efectividad, demostrada reiteradamente $(10,11)$. Por otro lado, se ha escrito que en los estudios adelantados sobre el sulfato ferroso desde el principio del siglo XIX, una de las principales constantes ha sido la presencia de efectos gastrointestinales secundarios, como acidez, dolor abdominal, náuseas y diarrea, lo que conlleva una disminución en la eficacia de las intervenciones (23). Así, se esperaría que al contrastar las reacciones adversas, estas estuvieran presentes en proporción diferente en el grupo que recibió sulfato ferroso, suposición que fue considerada en una de las hipótesis de esta investigación. Más de la tercera parte de los niños experimentó, al menos, una vez una de las reacciones adversas consideradas, proporción que fue más alta, en términos de frecuencias relativas, entre quienes tomaron hierro aminoquelado; no obstante, no se hallaron diferencias estadísticas. Una de las explicaciones posibles podría estar relacionada con el tamaño de la muestra, que pudiera no evidenciar diferencias cuando en realidad existen.

Al igual que en otras investigaciones, la elección del vehículo fue dispendiosa, como bien lo señalan Olivares y Pizarro (27); posiblemente, la tarea más difícil a la hora de fortificar un alimento con hierro es encontrar la combinación adecuada entre el compuesto y el vehículo. Para el caso específico de esta investigación, la leche pudo de alguna manera interactuar con los compuestos de hierro, siempre y cuando se ha reportado en la literatura científica que el calcio presente en la leche inhibe la absorción del hierro, tanto del heme como del no heme (22,28-36). Se eligió este vehículo debido a la inclusión de la leche en la minuta diaria de los niños, a su facilidad para la administración y a la oferta de licitaciones para la fortificación de leche con hierro en Colombia.

Una de las limitaciones, entre las cuales deben enmarcarse las conclusiones y hallazgos de este estudio, está relacionada con la muestra de investigación. Encontrar el número adecuado de preescolares con disminución de los niveles de hierro requería una inversión de tiempo y de recursos económicos considerables, que disminuía la viabilidad del proyecto, lo que derivó en que se trabajara con una muestra intencionada o por conveniencia, tanto por el tamaño como para la selección de preescolares.

Por otro lado, la dinámica de la población hizo difícil el seguimiento estricto de cada una de las unidades muestrales, presentándose al final del estudio ocho pérdidas correspondientes a niños que dejaron de asistir al centro infantil, principalmente por cambio en el domicilio.

Por último, y debido a la amplia discusión alrededor de la interacción entre el calcio y el hierro, los resultados de esta investigación deberán considerarse como una experiencia de fortificación de lecheenteracon hierro, de maneraque, al fortificar leche con hierro, se aconseja la utilización de hierro aminoquelado sobre el sulfato ferroso. En futuros estudios se podría estudiar el comportamiento del hierro aminoquelado en la fortificación de vehículos sin inhibidores o potenciadores de la absorción del hierro, comparándolos con la leche entera.

\section{Agradecimientos}

A la Fundación de Atención a la Niñez, en especial, a su nutricionista Viviana Ramírez, a las directoras y profesoras de los centros infantiles, y, por 
supuesto, a todos los niños que participaron en la investigación.

\section{Conflicto de interés}

Se indica un posible conflicto de interés en cuanto uno de los coinvestigadores es empleado de una de las entidades financiadoras del estudio.

\section{Financiación}

Esta investigación fue financiada por la Universidad CES y Nutreo S.A.S. Medellín, Antioquia.

\section{Referencias}

1. Freire WB. La anemia por deficiencia de hierro: estrategias de la OPS/OMS para combatirla. Salud Pública de México. 1998;40:199-205.

2. Olivares $\mathbf{G} \mathbf{M}$, Walter $\mathbf{K T}$. Consecuencias de la deficiencia de hierro. Rev Chil Nutr. 2003;30:226-33.

3. Badham J, Zimmermann JB, Kraemer K. Guía sobre anemia nutricional. Basilea: Sight and Life editores; 2007.

4. Organización Mundial de la Salud, Organización de las Naciones Unidas para la Agricultura y la Alimentación. Guidelines on food fortification with micronutrients. Fecha de consulta: 19 de marzo de 2012. Disponible en: http:// www.who.int/nutrition/publications/guide_food_fortification_ micronutrients.pd.

5. Agudelo-López S, Gómez-Rodríguez L, Coronado X, Orozco A, Valencia-Gutiérrez CA, Restrepo-Betancur LF, et al. Prevalencia de parasitosis intestinales y factores asociados en un corregimiento de la costa atlántica colombiana. Rev Salud Pública. 2008;10:633-42.

6. Organización de las Naciones Unidas para la Agricultura y la Alimentación. Procesamiento y fortificación de los alimentos. Fecha de consulta: 11 de enero de 2011. Disponible en: http://www.fao.org/docrep/006/w0073s/w0073s 10.htm.

7. Organización Mundial de la Salud. Anemia ferropénica. Informe de un grupo de estudio. Serie de informes técnicos número 182. Ginebra: OMS; 1959. p. 20.

8. Ministerio de la Protección Social, Instituto Colombiano de Bienestar Familiar, Instituto Nacional de Salud, Profamilia, Departamento Administrativo Nacional de Estadísticas. Encuesta Nacional de la Situación Nutricional en Colombia, 2010 (ENSIN). Bogotá: Da Vinci Editores; 2011.

9. Serna L, Aranceta J. Nutrición y salud pública: métodos, bases científicas y aplicaciones. Segunda edición. Barcelona: Masson S.A.; 2006.

10. Beinner M, Lamounier J. Recent experience with fortification of foods and beverages with iron for the control of iron-deficicncy anemia in Brazilian children. Food Nutr Bull. 2003;24:268-74.

11. Organización de las Naciones Unidas para la Agricultura y la Alimentación. Nutrición humana en el mundo en desarrollo. En: Carencia de hierro y otras anemias nutricionales. Fecha de consulta: 30 de diciembre de 2010. Disponible en: http://www.fao.org/docrep/006/w0073s/ w0073s0h.htm.
12. Moya A, Sevilla S. Estudio comparativo de hierro aminoquelado $V s$. sulfato ferroso más ácido fólico en el tratamiento de la anemia ferropénica en el embarazo. Revista Médica de los Post Grados de Medicina UNAH. 2008;11:42-9.

13. Fox T, Eagles J, Fairweather-Tait S. Bioavailability of iron glycine as a fortificant in infant foods. Am J Clin Nutr. 1998;67:664-8.

14. Madero D. Eficacia y seguridad del complejo de hidróxido de hierro férrico (III) y polimaltosa (IPC) Vs. el hierro aminoquelado en el manejo de la anemia ferropénica en niños. Rev AWGLA. 2007;1:28-9.

15. Instituto Colombiano de Bienestar Familiar. Encuesta Nacional de la Situación Nutricional en Colombia, 2005. Bogotá: ICBF; 2006.

16. Organización Mundial de la Salud, Departamento de Nutrición. WHO Anthro (version 3.2.2, January 2011) and macros. Fecha de consulta: 3 de junio de 2012. Disponible en: http://www.who.int/childgrowth/software/en/

17. UNICEF. El estado mundial de la infancia 1998. Tema: nutrición. Fecha de consulta: 6 de enero de 2011. Disponible en: http://www.unicef.org/spanish/sowc98sp/ silent.htm

18. Layrisse M, García-Casal M, Solano L, Baron M, Argüello F, Llovera D. Iron bioavailability in humans from breakfast enriched with iron bis-glycine chelate, phylates and polyphenols. J Nutr. 2000;130:2195-9.

19. Pineda O, Ashmead H, Pérez J, Lemus C. Effectiveness of iron amino acid chelate on the treatment of iron deficiency anemia in adolescents. J Appl Nutr. 1994;46:2-13.

20. Pineda O, Ashmead H. Effectinevess of treatment of iron deficiency anemia in infants and young children with ferrous bisglycinate chelate. Nutrition. 2001;17:381-4. http://dx.doi. org/10.1016/S0899-9007(01)00519-6,

21. Torres MA, Lobo NF, Sato K, Queiroz SS. Fortification of fluid milk for the prevention and treatment of iron defficiency anemia in children under 4 years of age. Revista de Saúde Pública. 1996;30:350-7.

22. Olivares M, Pizarro F, Pineda O, Name J, Hertrampf E, Walter T. Milk inhibits and ascorbic acid favors ferrous bis-glycine chelate bioavailability in humans. J Nutr. 1997; 127:1407-11.

23. Mayumi ÉC, Wander J, Bandeira J, Pereira E, Dichi I. Comparison of ferrous sulfate and ferrous glycinate chelate for the treatment of iron deficiency anemia in gastrectomized patients. Nutrition. 2008;24:663-8. http:// dx.doi.org/10.1016/j.nut.2008.03.017.

24. González-Rosendo G, Fernández-Ballart J, RodríguezJerez J, Sánchez-Muñoz J, Quintero-Gutiérrez A. Dosis semanal de hierro en mujeres adolescentes de Morelos (México). Ciencia y Tecnología Alimentaria. 2008;6:13742.

25. República de Colombia, Ministerio de la Protección Social. Resolución 2121 del 2010. Por la cual se adoptan los patrones de crecimiento publicados por la Organización Mundial de la Salud - OMS en el 2006 y 2007 para los niños, niñas y adolescentes de 0 y 18 años de edad y se dictan otras disposiciones. Res 2121/2010. Bogotá D.C.: Ministerio de la Protección Social; 2010. p. 51. 
26. Fundación de Atención a la Niñez. Fecha de consulta: 3 de noviembre de 2011. Disponible en: http://www.fan.org. co/detalles-novedosos/objeto-social.html

27. Olivares GM, Pizarro F. Bioavailability of iron bis-glycinate chelate in water. Arch Latinoam Nutr. 2001;51:22-8.

28. Barton J, Conrad M, Parmley R. Calcium inhibition of inorganic iron absorption in rats. Gastroenterology. 1983;84:90-101.

29. Hallberg L, Brune M, Erlandsson M, Sandberg A, Rossander-Hulten L. Calcium: Effect of different amounts on nonheme- and hemeiron absorption in humans. Am J Clin Nutr. 1991;53:112-9.

30. Monsen E, Cook J. Food iron absorption in human subjects. IV. The effects of calcium and phosphorus salts on the absorption of nonheme iron. Am J Clin Nutr. 1976;29:1142-8.

31. CookJ, DassenkoS, Whittaker P. Calciumsupplementation: Effect on iron absorption. Am J Clin Nutr. 1991;53:106-11.
32. Reddy M, Cook J. Effect of calcium intake on nonhemeiron absorption from a complete diet. Am J Clin Nutr. 1997;65:1820-5.

33. Hallberg L, Rossander-Hulten L, Brune M, Gleerup A. Calcium and iron absorption: Mechanism of action and nutritional importance. Eur J Clin Nutr. 1992;46:317-27.

34. Hallberg L. Does calcium interfere with iron absorption? Am J Clin Nutr. 1998;68:3-4.

35. Hallberg L, Rossander-Hulten L, Brune M, Gleerup A. Inhibition of haem-iron absorption in man by calcium. $\mathrm{Br} J$ Nutr. 1992;69:533-40. http://dx.doi.org/10.1079/ BJN19930053

36. Alcaraz G, Bernal C, Aristizábal M, Ruiz M, Fox J. Anemia y anemia por déficit de hierro en niños menores de cinco años y su relación con el consumo de hierro en la alimentación. Turbo, Antioquia, Colombia. Invest Educ Enferm. 2006;24:16-29. 